\title{
Flash co-pyrolysis of biomass: The influence of biopolymers
}

\author{
T. Cornelissen ${ }^{\text {a }}$, M. Jans ${ }^{\text {d }}$, M. Stals ${ }^{\text {a }}$, T. Kuppens ${ }^{\text {b }}$, T. Thewys $^{\text {b }}$, G.K. Janssens ${ }^{c}$, \\ H. Pastijn ${ }^{\text {e }}$, J. Yperman ${ }^{\text {a,* }}$, G. Reggers ${ }^{\text {a }}$, S. Schreurs ${ }^{d}$, R. Carleer ${ }^{\text {a }}$ \\ a Lab of Applied and Analytical Chemistry, CMK, Hasselt University, Agoralaan Gebouw D, 3590 Diepenbeek, Belgium \\ ${ }^{\mathrm{b}}$ Economics and Law, CMK, Hasselt University, Agoralaan Gebouw D, 3590 Diepenbeek, Belgium \\ ' Operations Management, Hasselt University, Agoralaan Gebouw D, 3590 Diepenbeek, Belgium \\ ${ }^{\mathrm{d}} \mathrm{NuTeC}$, department IWT, XIOS, Agoralaan Gebouw H, 3590 Diepenbeek, Belgium \\ e Department of Mathematics, Royal Military Academy, Renaissance Avenue 30, 1000 Brussels, Belgium
}

A R T I C L E I N F O

\section{Article history:}

Received 30 June 2008

Accepted 1 December 2008

Available online 6 December 2008

\section{Keywords:}

Flash co-pyrolysis

Willow

Biopolymer

Pyrolytic water

Bio-oil

Chemicals

Upgrading

Waste

Synergy

MCDA

MCDM

PROMETHEE

\begin{abstract}
A B S T R A C T
A high water content is one of the major drawbacks for the utilisation of bio-oil. One technology which shows the potential to satisfy the demand for bio-oil with a reduced water content is the flash copyrolysis of biomass with biopolymers. The influence of biopolymers on the pyrolysis yield of a biomass waste stream is investigated with a semi-continuous home-built pyrolysis reactor. Polylactic acid (PLA), corn starch, polyhydroxybutyrate (PHB), Biopearls, Eastar, Solanyl and potato starch are the biopolymers under investigation. All biopolymers show their specific benefits during flash co-pyrolysis with willow (target biomass) at $723 \mathrm{~K}$. Each (co-)pyrolysis of pure willow (reference) and all 1:1 (w/w) ratio willow/ biopolymer blends is evaluated based on five predefined criteria. A multi-criteria decision aid (MCDA) method 'PROMETHEE' is used in order to obtain an objective ranking of the different biopolymer options.

The flash co-pyrolysis of biomass and biopolymers results in improved pyrolysis characteristics. The flash co-pyrolysis of $1: 1$ willow/PHB is the most performant option, while $1: 1$ willow/PLA, 1:1 willow/ Biopearls and 1:1 willow/potato starch show increased potential as well. The fact that biopolymers, despite their biodegradability, should be considered as waste, further increases the appealing features of the flash co-pyrolysis of biomass and biopolymers.
\end{abstract}

(c) 2008 Elsevier B.V. All rights reserved.

\section{Introduction}

Part of the current research programs world-wide is focussing on the evolution of energy and materials to become sustainable. The flash pyrolysis of biomass waste streams is a promising method to obtain both energy and materials (e.g. chemicals), without endangering the basic food supply chain. Pyrolysis is an anaerobic thermal degradation process in which biomass is converted to (bio-)char, (bio-)gas and bio-oil [1-3]. All obtained pyrolysis products are suitable for use as energy feedstock. Nevertheless, bio-oil shows the most potential and can substitute fuel oil or diesel in many static applications. Additionally, it can be upgraded to become a transportation fuel [4]. Also bio-oil is a source of value-added chemicals [5,6]. However, it often contains up to 300 different compounds [7], making it cumbersome and economically unattractive to isolate chemicals from. A major

\footnotetext{
* Corresponding author. Tel.: +32 11268 320; fax: +32 11268301.

E-mail address: jan.yperman@uhasselt.be (J. Yperman).
}

drawback of (flash) pyrolysis of biomass is the inherent production of pyrolytic water, which results in a bio-oil with a relatively high water content. In view of the applicability of bio-oil in general, water is defined as detrimental [8].

Flash co-pyrolysis results in enhanced pyrolysis characteristics. The flash co-pyrolysis of willow and the biopolymers polylactic acid (PLA) and polyhydroxybutyrate (PHB), respectively, induce interactions resulting in a synergetic decrease in the amount of pyrolytic water $[9,10]$. Additionally, a synergetic increase in pyrolysis yield and in energy recuperation is obtained. The synergetic effect is observed to increase along with the addition of PLA and PHB, respectively. Besides bio-oil, the flash co-pyrolysis of willow and PHB results in the production of crystals of crotonic acid, which offer added value as a source of chemicals [10]. Even though biopolymers originate from renewables and/or are biologically degradable, most of them still have to be considered as waste, since it is ecologically unacceptable to dispose of them in the environment. Therefore, the flash co-pyrolysis of biomass and biopolymers is a promising route, not only as a supplier of renewable energy, but also as an attractive upgrading method for 
the pyrolysis of biomass, as a supplier of value-added materials, and as an alternative waste treatment option.

A myriad of biopolymers (PLA, corn starch, PHB, Biopearls, Eastar, Solanyl and potato starch) has been investigated in identical circumstances to evaluate their influence on the flash co-pyrolysis behaviour of uncontaminated willow (target biomass) at $723 \mathrm{~K}$ with a semi-continuous home-built pyrolysis reactor. The flash pyrolysis of pure (100\%) uncontaminated willow serves as a reference. However, future experiments will be directed towards the flash (co-)pyrolysis of willow contaminated with heavy metals, originating from phytoremediation fields $[11,12]$.

Based on five predefined criteria (e.g. water content, water-free bio-oil yield, etc.), the different flash (co-)pyrolysis experiments are compared with a multi-criteria decision aid (MCDA) methodology 'PROMETHEE'. PROMETHEE, which belongs to the outranking family of MCDA methodologies, is implemented by the 'Decision Lab' tool. This decision support tool is comparing pairwise the different biopolymer options and ranks them, based on assessments of these options for the different criteria [13-15]. The respective importance of the predefined criteria is expressed by weights which are computed by means of pairwise comparisons of the criteria and 'MCDM tool', a software that incorporates an eigenvalue method, related to the Analytic Hierarchy Process (AHP) method by Saaty [16].

\section{Experimental}

\subsection{Materials}

The experiments are performed on uncontaminated willowSalix, and seven different biopolymers. The biopolymers, which are a special kind of plastic, originate from renewables and/or are biologically degradable. Despite their biodegradability, however, most biopolymers still have to be considered as waste, since it would be ecologically unacceptable to dispose of them in the environment [17]. Based on their origin, biopolymers can be classified into two main categories: biopolymers produced on the basis of renewable feedstocks and biopolymers with biodegradable characteristics but manufactured from petrochemical resources. The physical and chemical structures of the biopolymers are the basic properties that affect the degradation and the biodegradation [18]. De Schoenmakere [17] notes that not all biodegradable materials are compostable and that not all compostable materials have a biological origin.

The renewable biopolymers can be subdivided into three groups $[17,19]$ :

- Biopolymers directly obtained from biomass: e.g. cellulose, proteins, fats and polysaccharides. Three different biopolymers that belong to the group of polysaccharides, and more specifically the subgroup of starch-based biopolymers, are considered for this research: e.g. corn starch, potato starch and Solanyl.

- Biopolymers directly produced by micro-organisms: e.g. cellulose, polysaccharides and polyhydroxyalkanoates (PHA's). Polyhydroxybutyrate, an aliphatic polyester, is the most applied PHA. PHB can be produced by bacteria, yeasts, and/or plants $[17,20]$.

- Biopolymers formed via chemical synthesis: e.g. polyesters. For this research polylactic acid is investigated. PLA is obtained by polymerisation of the renewable fermentation product lactic acid. Lactic acid can be synthesised by chemical means, but is generally produced by the microbial fermentation of sugars and starch $[17,21]$. PLA shows to be one of the worst biodegradable biopolymers $[17,18,20]$.

Biopearls is additionally investigated. Biopearls stems from plant material $[22,23]$. However, the exact origin of this biopolymer is unknown to the authors. Finally, EastarBio is chosen as a synthetic biopolymer. EastarBio is a polytetramethyleneadipate-terephthalate and belongs to the modified polyethylene terephthalates (PET).

The beforementioned biopolymers are evaluated as potential pyrolysis "enhancers". Therefore, the biopolymers are co-pyrolysed with willow in a 1:1 (w/w)-ratio. The willow branches (the leaves are not taken into account in this study) and all biopolymers are shredded into small particles $(<2 \mathrm{~mm})$ to ensure flash pyrolysis of the entire particle. The experiments on willow/biopolymer blends with a w/w-ratio of $1: 1$ are investigated and compared with the results obtained for $100 \%$ pure willow (=reference sample) to evaluate the effects on the flash co-pyrolysis behaviour.

\subsection{Flash (co-)pyrolysis}

The semi-continuous home-built pyrolysis reactor, injection and recuperation system, Fig. 1 part a, b and c, respectively, are manufactured in stainless steel (AISI 304). The entire system is continuously flushed with nitrogen gas in order to guarantee an oxygen-deficient environment. Within the reactor, a heat transfer medium (white sand) is in constant motion with the aid of an Archimedical screw. The reactor is heated by a tailored heating jacket.

As soon as the sand inside the reactor reaches the pyrolysis temperature ( $\mathrm{PT}=723 \mathrm{~K}$ ), the nitrogen flow is stopped and the injection system is started; inserting the willow or willow/ biopolymer blend into the reactor. The willow or willow/ biopolymer blend subsequently undergoes flash (co-)pyrolysis and is converted into volatiles which mainly condense into the recuperation system as condensables. The term "condensables", which represents the pyrolysis yield, is used to group bio-oil and crystals. An extended pyrolysis flowchart (Fig. 2) is added to represent the observations of this research. The occurrence of crystals is only observed after the flash co-pyrolysis of willow and PHB, and is discussed in more detail in Ref. [10]. A flowchart of the flash (co-)pyrolysis is schematically presented in Fig. 2. For a detailed description of the experimental flash pyrolysis set-up and procedure, please refer to Ref. [9].

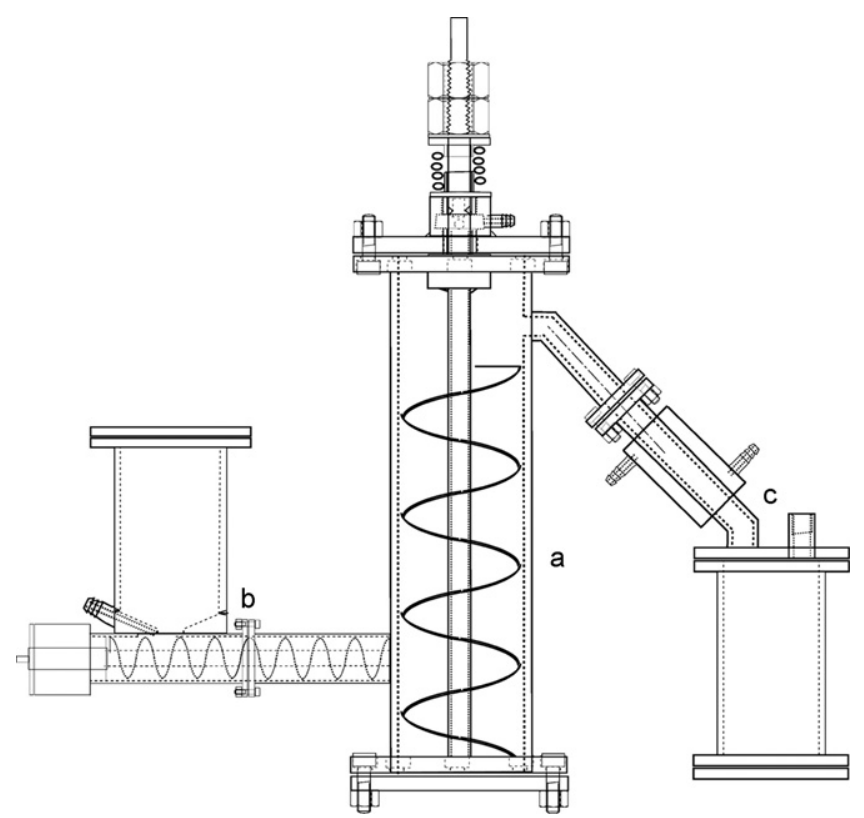

Fig. 1. Pyrolysis set-up: (a) reactor (at $723 \mathrm{~K}$ ); (b) injection system (at RT in K) with a biomass reservoir; and (c) recuperation system (at RT in $\mathrm{K}$ ) with an additional water cooler [9]. 


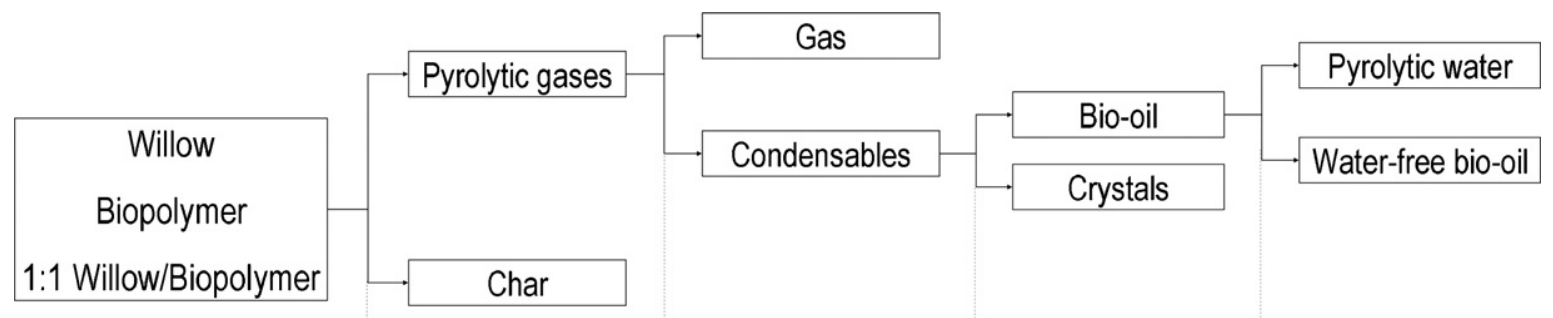

Flash (co-)Pyrolysis (723K) $\quad$ Cooling (RT in K) $\quad$ Cnystalisation \& Filtration $\quad$ Virtual separation (water content in \%)

Fig. 2. Extended schematic representation of the flash (co-)pyrolysis flowchart [10].

\subsection{Analysis}

\subsubsection{Ultimate analysis}

The CHNS- and O-content of the input materials (willow and biopolymers) are determined via two distinct experiments, using a FlashEA 1112 Elemental Analyser of Thermo Electron Corporation. Approximately 2-4 mg of sample is introduced into a container, which is injected by an autosampler. In case of CHNS-determinations, 5-10 mg vanadiumpentoxide is added to the sample as a combustion catalyst. 2,5-Bis(5-tert-butyl-benzoxazol-2-yl)thiophene (BBOT- $\mathrm{C}_{26} \mathrm{H}_{26} \mathrm{~N}_{2} \mathrm{O}_{2} \mathrm{~S}$ ) and L-cystine $\left(\mathrm{C}_{6} \mathrm{H}_{12} \mathrm{~N}_{2} \mathrm{O}_{4} \mathrm{~S}_{2}\right)$ are used as standards for the CHNS- and O-determinations, respectively.

\subsubsection{Thermogravimetric analysis (TGA)}

About $30 \mathrm{mg}$ of sample is pyrolysed under approximately $35 \mathrm{ml} / \mathrm{min} \mathrm{N}_{2}$ flow at a heating rate of $10 \mathrm{~K} / \mathrm{min}$ from room temperature (RT) to $973 \mathrm{~K}$ with a DuPont Instruments 951 Thermogravimetric Analyzer.

\subsubsection{Calorimetry}

The higher heating values (H.H.V.'s) of the input materials (willow and biopolymers) and of the pyrolysis products (bio-oil and crystals) are determined with the aid of an IKA C5003 control calorimeter equipped with an IKA KV 600 Digital water cooler and a Sartorius CP224S analytical balance. Oxygen is connected to the system to pressurise the bomb. Measurements are executed in dynamic mode and the calibration of the system is performed with benzoic acid (palleted, C723) of IKA with a higher heating value of $26.5 \mathrm{MJ} / \mathrm{kg}$.

\subsubsection{Water content}

Due to the relatively high percentages of water, the water content of the different samples is measured using the Dean-Stark method (also see Section 4). Around 5-10 ml sample is introduced into a $250 \mathrm{ml}$ flask together with approximately $60 \mathrm{ml}$ toluene. Water is separated from the sample in azeotropic conditions into a calibrated reservoir, indicating the amount of water present in the sample.

\subsubsection{Gas chromatography/mass spectrometry (GC/MS)}

A Varian 3400 gas chromatograph is equipped with a Finnigan TSQ 700 mass selective detector and a DB-WAX capillary fused silica column $(30 \mathrm{~m} \times 0.25 \mathrm{~mm} \times 0.25 \mu \mathrm{m})$. The column temperature is programmed from 308 to $533 \mathrm{~K}$ at $12 \mathrm{~K} / \mathrm{min}$ after an initial $1 \mathrm{~min}$ isothermal period and kept at the final temperature for $6 \mathrm{~min}$. Sample injection of $1 \mu \mathrm{l}$ of a $1-4 \%$ solution in methanol is performed in the splitless mode with the injector temperature set at $533 \mathrm{~K}$.

The mass spectrometer is set at the standard ionising voltage of $70 \mathrm{eV}$ with a mass range $\mathrm{m} / z$ of $35-500$ and a scan rate of 2 scans/s. The identification of the compounds is accomplished by a library search in a NIST database.

\section{Results and discussion}

The main characteristics of willow and the seven biopolymers are listed in Table 1. All materials have a high C-and O-content, an intermediate $\mathrm{H}$-content, and a very low to negligible $\mathrm{N}$-content. No sulphur is detected. The calorific values of the input materials vary between 17.0 and $26.2 \mathrm{MJ} / \mathrm{kg}$.

For the slow pyrolysis $(10 \mathrm{~K} / \mathrm{min}$ ) of the pure materials, the mass loss curves and the derivatives, obtained by TGA, are shown in Fig. 3. Some basic characteristics, as summarised in Table 1, can be deduced. Additionally, a first indication of the general pyrolysis behaviour of the input materials is obtained. Willow (Fig. 3a) decomposes within a relatively wide temperature interval. The main

Table 1

Main characteristics (on dry basis) of willow and the biopolymers.

\begin{tabular}{|c|c|c|c|c|c|c|c|c|}
\hline Characteristics & Willow & PLA & Corn starch & PHB & Biopearls & Eastar & Solanyl & Potato starch \\
\hline \multicolumn{9}{|c|}{ Proximate analysis (\%) } \\
\hline Moisture & 1.88 & 0.00 & 0.48 & 0.17 & 0.00 & 0.00 & 1.05 & 0.00 \\
\hline Volatile & 75.27 & 98.05 & 92.78 & 97.97 & 94.92 & 95.02 & 80.81 & 87.56 \\
\hline Fixed C & 21.14 & 1.30 & 4.62 & 0.80 & 1.77 & 3.68 & 13.00 & 3.87 \\
\hline Ash & 1.71 & 0.65 & 2.12 & 1.06 & 3.31 & 1.30 & 5.14 & 8.57 \\
\hline \multicolumn{9}{|c|}{ Ultimate analysis (\%) } \\
\hline Carbon & 46.91 & 49.84 & 56.21 & 55.96 & 52.85 & 62.45 & 43.96 & 49.76 \\
\hline Hydrogen & 5.95 & 5.63 & 6.89 & 7.07 & 6.01 & 7.07 & 7.21 & 5.65 \\
\hline Nitrogen & 0.63 & 0.15 & 0.18 & 0.14 & 0.00 & 0.03 & 0.10 & 0.10 \\
\hline Oxygen & 41.69 & 44.42 & 36.77 & 36.40 & 33.15 & 31.85 & 50.00 & 39.33 \\
\hline $\mathrm{H} / \mathrm{C}$ molar ratio & 1.52 & 1.36 & 1.47 & 1.52 & 1.36 & 1.36 & 1.97 & 1.36 \\
\hline $\mathrm{O} / \mathrm{C}$ molar ratio & 0.67 & 0.67 & 0.49 & 0.49 & 0.47 & 0.38 & 0.85 & 0.59 \\
\hline \multicolumn{9}{|c|}{ Calorimetric analysis } \\
\hline H.H.V. (MJ/kg) & 18.7 & 18.6 & 22.9 & 22.8 & 20.5 & 26.2 & 17.0 & 18.9 \\
\hline
\end{tabular}



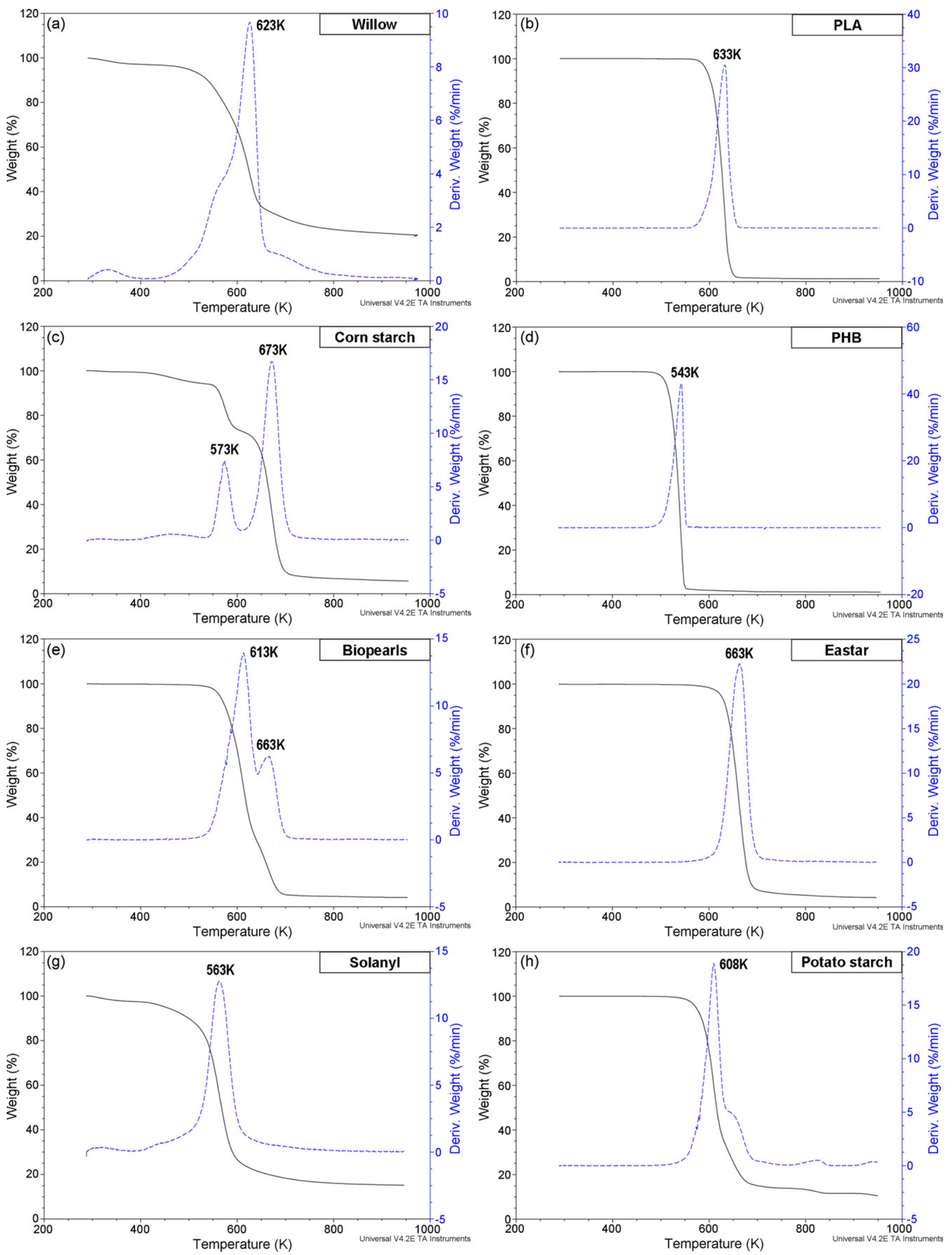

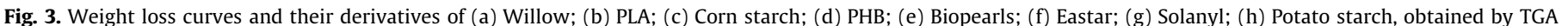
(program: RT $\rightarrow 973 \mathrm{~K}$ at $10 \mathrm{~K} / \mathrm{min}$ ). 
Table 2a

Pyrolysis yields and efficiencies of the condensables, char and gas productions for the different biopolymer options.

\begin{tabular}{|c|c|c|c|c|c|c|c|c|}
\hline & Willow & Willow/PLA & Willow/corn starch & Willow/PHB & Willow/Biopearls & Willow/Eastar & Willow/Solanyl & Willow/potato starch \\
\hline \multicolumn{9}{|l|}{ Input (m\%) } \\
\hline Willow & 100.00 & 51.80 & 50.36 & 50.06 & 49.90 & 49.83 & 52.57 & 49.97 \\
\hline Biopolymer & 0.00 & 48.20 & 49.64 & 49.94 & 50.10 & 50.17 & 47.43 & 50.03 \\
\hline \multicolumn{9}{|l|}{ Output (m\%) } \\
\hline Condensables & 50.10 & 51.96 & 43.72 & 64.24 & 52.79 & 50.01 & 59.24 & 51.52 \\
\hline Char & 22.39 & 13.46 & 14.47 & 9.50 & 12.92 & 13.92 & 15.24 & 13.49 \\
\hline Gases (by diff.) & 27.50 & 34.58 & 41.81 & 26.26 & 34.29 & 36.07 & 25.52 & 34.99 \\
\hline
\end{tabular}

a Input $(\mathrm{m} \%)$ is calculated on dry basis.

Table 2b

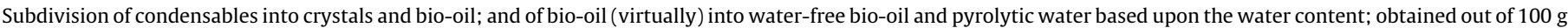
input.

\begin{tabular}{|c|c|c|c|c|c|c|c|c|}
\hline & Willow & Willow/PLA & Willow/corn starch & Willow/PHB & Willow/Biopearls & Willow/Eastar & Willow/Solanyl & Willow/potato starch \\
\hline Cristals (g) & 0.00 & 0.00 & 0.00 & 29.70 & 0.00 & 0.00 & 0.00 & 0.00 \\
\hline Bio-oil (g) & 50.10 & 51.96 & 43.72 & 34.54 & 52.79 & 50.01 & 59.24 & 51.52 \\
\hline Water content (m\%) & 36.65 & 15.53 & 26.94 & 15.97 & 16.81 & 18.96 & 32.82 & 16.17 \\
\hline Water-free Bio-oil (g) & 31.74 & 43.89 & 31.94 & 29.03 & 43.92 & 40.53 & 39.80 & 43.19 \\
\hline Pyrolytic Water (g) & 18.36 & 8.07 & 11.78 & 5.52 & 8.87 & 9.48 & 19.44 & 8.33 \\
\hline
\end{tabular}

decomposition of willow takes place in the range of 473-673 K. Some biopolymers on the other hand (PLA, PHB, Eastar and Solanyl; Fig. 3b, d, f and g, respectively) decompose in one single and narrow temperature interval, while others show a multi-step decomposition (corn starch, Biopearls and potato starch; Fig. 3c, e and h, respectively). As compared with willow $\left(T_{\max }=623 \mathrm{~K}\right)$, the maximum degradation temperature $\left(T_{\max }\right)$ of some biopolymers (PLA and Eastar) is higher, while for others (PHB, Solanyl and potato starch) $T_{\max }$ is lower. In the case of corn starch and Biopearls, however, the comparison of $T_{\max }$ is less straightforward.

\subsection{Flash co-pyrolysis}

To study the influence of the biopolymers on the pyrolysis behaviour of willow, the flash (co-)pyrolysis of each biopolymer option (pure willow, and 1:1 willow/biopolymer blends) is performed at $723 \mathrm{~K}$ with the pyrolysis reactor shown in Fig. 1. Besides bio-oil, the flash co-pyrolysis of willow and PHB results in the production of crystals of crotonic acid (Fig. 2 shows the extended pyrolysis flowchart). These crystals appear after a spontaneous phase separation in the recuperation system (Fig. 1, part c) and offer added value as a source of chemicals. Table 2a outlines a brief summary of the pyrolysis yields and efficiencies of the condensables (total amount of bio-oil and crystals), char and gas productions. The amount of gases is calculated by difference. In Table $2 \mathrm{~b}$, a subdivision of condensables in bio-oil and crystals and of bio-oil (virtually) in water-free bio-oil and pyrolytic water, based on the water content, is shown. From both tables, it can be concluded that not all biopolymers react in the same way during flash co-pyrolysis with willow.
In Table 2a, a first indication of the effect of biopolymers is observed: the flash co-pyrolysis of willow and biopolymers results in a reduced char yield compared with the willow reference and, except for the biopolymers corn starch and Eastar, a higher yield in condensables is achieved. Table $2 b$ shows that, generally, a significant reduction of the water content is obtained via flash co-pyrolysis of willow with biopolymers. Except for Solanyl, a vast reduction of the total amount of pyrolytic water is observed. Finally, all biopolymer options, except for PHB, result in an enhanced water-free bio-oil yield.

The averaged experimental H.H.V.'s of the different bio-oils are summarised in Table 2c. The addition of biopolymers (except for Solanyl) clearly shows an additional advantage: an increase in the H.H.V. of bio-oil produced from willow/biopolymer blends compared to the bio-oil of pure willow (16.1-20.8 MJ/kg). Taking into account the yield in condensables (Tables $2 \mathrm{a}$ and $2 \mathrm{~b}$ ) and the experimental H.H.V. of the input materials (Table 1), of the respective bio-oil (Table 2c) and of the crystals (H.H.V. of crystals $=23.1 \mathrm{MJ} / \mathrm{kg}$ ), the energy recuperation for each biopolymer option can be calculated as follows:

$$
\begin{aligned}
& \text { Energy recuperation in condensables } \\
& =\frac{\left[\text { bio-oil } \times \mathrm{HHV}_{\text {bio-oil }}+\text { crystals } \times \mathrm{HHV}_{\text {crystals }}\right]}{\left[\text { biomass } \times \mathrm{HHV}_{\text {biomass }}+\text { biopolymer } \times \mathrm{HHV}_{\text {biopolymer }}\right]} \times 100
\end{aligned}
$$

For instance, the energy recuperation in condensables for the $1: 1$ willow/PLA flash co-pyrolysis is:

$$
=\frac{51.96 \mathrm{~g} \times 18.5 \mathrm{~kJ} / \mathrm{g}+0.00 \mathrm{~g} \times 23.1 \mathrm{~kJ} / \mathrm{g}}{51.80 \mathrm{~g} \times 18.7 \mathrm{~kJ} / \mathrm{g}+48.20 \mathrm{~g} \times 18.6 \mathrm{~kJ} / \mathrm{g}} \times 100=51.54 \%
$$

\begin{tabular}{|c|c|c|c|c|c|c|c|c|}
\hline & Willow & Willow/PLA & Willow/corn starch & Willow/PHB & Willow/Biopearls & Willow/Eastar & Willow/Solanyl & Willow/potato starch \\
\hline H.H.V. (MJ/kg) & 16.1 & 18.5 & 18.5 & 20.2 & 19.1 & 20.8 & 15.7 & 19.2 \\
\hline Energy recuperation (\%) & 43.1 & 51.5 & 38.9 & 66.8 & 51.5 & 46.3 & 52.1 & 52.6 \\
\hline
\end{tabular}

Table 2c

H.H.V.'s of the bio-oils in Mega Joule per kilogram and energy recuperation of the biopolymer options in \%a.

\footnotetext{
a The energy recuperation of willow $/ \mathrm{PHB}=$ sum of bio-oil and crystals (H.H.V. $=23.1 \mathrm{MJ} / \mathrm{kg}$ ).
} 
Table 3

The influence of biopolymers on the amount of pyrolytic water produced.

\begin{tabular}{lccc}
\hline Biopolymer option & \multicolumn{2}{c}{ Pyrolytic water (m\%) } & \\
\cline { 2 - 4 } & Actual amount & Absolute minimum & Influence (\%) \\
\hline Willow & 18.36 & 18.36 & - \\
Willow/PLA & 8.07 & 9.51 & -15 \\
Willow/corn starch & 11.78 & 9.25 & +27 \\
Willow/PHB & 5.52 & 9.19 & -40 \\
Willow/Biopearls & 8.87 & 9.16 & -3 \\
Willow/Eastar & 9.48 & 9.15 & +4 \\
Willow/Solanyl & 19.44 & 9.65 & +101 \\
Willow/potato starch & 8.33 & 9.18 & -9 \\
\hline
\end{tabular}

The biopolymer options in italic result in a synergy.

Except for 1:1 willow/corn starch, all biopolymer options result in an enhanced energy recuperation compared with the willow reference.

Additionally, it should also be emphasised that some biopolymers result in a synergy during the flash co-pyrolysis with willow. This synergy is best illustrated by calculating the difference between the actual amount and the minimum amount of pyrolytic water produced during the flash co-pyrolysis. The actual amount of pyrolytic water is calculated via the bio-oil yield and the respective water content (Table 2). The crystals, originating from the flash co-pyrolysis of willow and PHB, do not contain water. The minimum amount of pyrolytic water, for which it is assumed that the biopolymer itself does not result in the formation of pyrolytic water during flash pyrolysis (=absolute minimum), is calculated by the amount of willow used in the respective blend and the pyrolysis results of pure willow (reference): $100 \mathrm{~g}$ willow results in $18.36 \mathrm{~g}$ pyrolytic water (Table 2b). Table 3 summarises the influence of each biopolymer on the production of pyrolytic water during flash co-pyrolysis with willow. Four biopolymer options result in a lower actual amount of pyrolytic water compared with the absolute minimum amount and thus result in a synergy: PHB, PLA, potato starch and Biopearls. The flash co-pyrolysis of $1: 1$ willow/PHB shows the highest synergy, reaching minus $40 \%$ [10]: the absolute minimum amount of pyrolytic water for willow/PHB $=[(50.06 \mathrm{~g} \times 18.36 \%+$ $49.94 \mathrm{~g} \times 0.00 \%) / 100]=9.19 \mathrm{~m} \%$, while the actual yield in pyrolyitc water is $5.52 \mathrm{~m} \%$ (Table $2 \mathrm{~b}$ ), thus a decrease of $3.67 \mathrm{~m} \%$ or [(3.67/ $9.19) \times 100]=-39.93 \%$. The other three biopolymers (corn starch, Eastar and Solanyl) do not provide any straightforward evidence towards the occurrence of such a synergy.

Unfortunately, there is not one specific biopolymer option that is dominant in all areas of interest. For instance, the willow/Solanyl blend results in the highest bio-oil yield, but shows the lowest reduction in water content and results in a bio-oil with the lowest H.H.V. as compared with the other willow/biopolymer blends. Additionally, not all areas of interest are of equal importance, making it ambiguous to decide which biopolymer option is the best one to pursue. This is a typical example of a multi-criteria decision making problem.

\subsection{Multi-criteria decision making}

In order to determine which biopolymer option performs best, the multi-criteria decision aid software 'Decision Lab 2000Executive Edition, Version 1.0' of Visual Decision Inc., based on PROMETHEE and GAIA, is applied [15]. PROMETHEE and GAIA belong to a family of multi-criteria decision aid methods known as outranking methods and are based on the principle of pairwise comparison [13-15]. Some different steps need to be carried out to correctly model and analyse the MCDM problem with Decision Lab: defining the actions (=biopolymer options), defining the criteria, granting each criterion an objective weight, constructing an evaluation table, choosing a transformation function and respective thresholds for each criterion, and finally analysing the MCDM problem.

Eight different biopolymer options are under investigation: the flash pyrolysis of pure willow, which serves as a reference, and the flash co-pyrolysis of willow and polylactic acid, corn starch, polyhydroxybutyrate, Biopearls, Eastar, Solanyl and potato starch, respectively, all in a w/w-ratio of $1: 1$. Each biopolymer option is evaluated using five predefined criteria: (1) water-free bio-oil yield, (2) water content, (3) energy recuperation, (4) char yield and (5) total amount of readily separable chemicals. Because all criteria differ in importance, the respective weights of the criteria must be taken into account. To grant each criterion an acceptable weight, a decision support software 'MCDM tool 1.0, beta version', which incorporates an eigenvalue method related to the Analytic Hierarchy Process method by Saaty, is applied [16,24]. A $5 \times 5$ pairwise comparison matrix of the relative importance of the five predefined criteria, interpreted by and based upon the experience and judgment of some of the authors, is constructed (Matrix 1 ). The entry in row $i$ and column $j$ of Matrix 1 indicates how much more important criterion $i$ is than criterion $j$. "Importance" is measured on an integer-valued 1-9 ratio scale and its respective reciprocals [16].

To obtain Matrix 1, the following motivation is applied. The amount of readily separable chemicals is fairly the most important criterion because separated chemicals have a much higher economic importance than energy. The water-free bio-oil yield is considered the second most important criterion, because it summarises the valorisable fraction of the bio-oil that has the potential for application as an energy source and, in the future, as a source of value-added chemicals which are still dissolved in the bio-oil. The criterion 'Energy recuperation' only focuses on the valorisable fraction of the bio-oil as energy source, which makes it economically less attractive than the water-free bio-oil yield. Even though the water content is a very important aspect of bio-oil, it is conceived as the least but one important criterion under consideration since it only provides an indication towards the applicability and usability of the bio-oil. Finally, the char yield, which is only considered as a by-product for this research, in particular when contaminated biomass is pyrolysed, is selected as the least important criterion. With the aid of MCDM tool, the normalised principal eigenvector of Matrix 1 is calculated, resulting in the respective weights of each criterion. Table 4 ranks the criteria according to their relative weights (sum equals ' 1 ') obtained by MCDM tool and summarises the motivation applied. Additionally, MCDM tool calculates a consistency ratio (C.R.) of 0.006. In a perfectly consistent comparison matrix, a C.R. of zero would be obtained. This, however, is very unlikely and some inconsistency is allowed (consistent if: C.R. $<0.1$ ). If C.R. is higher than 0.1 , a revision of the pairwise comparisons would be required [16].

Once all data have been gathered, an evaluation table is constructed (Table 5). Each criterion corresponds to a column and each biopolymer option to a row. In the top of the evaluation table, some characteristic parameters of the respective criteria are listed. The maximisation or the minimisation requirement for each criterion is one of the crucial criterion qualifiers [15]. It is supposed to be advantageous if the water-free bio-oil yield, the energy recuperation, and the total amount of readily separable chemicals are high; while the water content and the char yield are low. Additionally, PROMETHEE (Decision Lab) offers six transformation functions for six different types of criteria: Usual (e.g. qualitative data), U-shape (e.g. discrete resources), V-shape (e.g. operational criteria), Level (e.g. financial long term), Linear (e.g. financial short term) and Gaussian (e.g. security). Each function is defined by 
Table 4

Summary of the five predefined criteria ranked according to their relative weights and the motivation applied.

\begin{tabular}{|c|c|c|}
\hline Criterion & Weight & Motivation \\
\hline Total amount of readily separable chemicals & 0.4543 & The economic value of separated chemicals is higher than energy \\
\hline Water-free bio-oil yield & 0.2511 & The valorisable fraction: energy and dissolved chemicals \\
\hline Energy recuperation & 0.1525 & The valorisable fraction: energy only \\
\hline Water content & 0.0887 & An indication for the applicability of the bio-oil \\
\hline Char yield & 0.0533 & A by-product \\
\hline
\end{tabular}

Matrix 1: Pairwise comparison matrix $(5 \times 5)$ to obtain the respective weights of each criterion.

\begin{tabular}{|c|c|c|c|c|c|c|}
\hline & & 1 & 2 & 3 & 4 & 5 \\
\hline Water-free bio-oil yield & 1 & 1 & 3 & 2 & 4 & $1 / 2$ \\
\hline Water content & 2 & $1 / 3$ & 1 & $1 / 2$ & 2 & $1 / 5$ \\
\hline Energy recuperation & 3 & $1 / 2$ & 2 & 1 & 3 & $1 / 3$ \\
\hline Char yield & 4 & $1 / 4$ & $1 / 2$ & $1 / 3$ & 1 & $1 / 8$ \\
\hline Total amount of readily separable chemicals & 5 & 2 & 5 & 3 & 8 & 1 \\
\hline
\end{tabular}

parameters like an indifference threshold $q$, a preference threshold $p$, and a Gaussian threshold $s$. When a transformation function and the respective threshold values have been defined for each criterion, the deviations between the evaluations of pairs of biopolymer options on one criterion are translated into a preference degree $P_{i}(a, b)$, with $a$ and $b$ representing two different biopolymer options, ranging between 0 and 1 , allowing a comparison of one criterion with another, independently from the scales of measurement.

The preference degree is a non-decreasing function of the deviation: smaller deviations will contribute to weaker degrees of preference, and larger ones to stronger degrees of preference. For a detailed description of the transformation functions and the thresholds, please refer to Refs. [13-15]. The choice of such a transformation function and the respective thresholds is first and foremost directed by the guideline: minimise the loss of information during transformation. This can be translated into the following rule: the more accurate the data, the more continuous the transformation function. For this research, the Vshape transformation function is applied the most because it fits the characteristics of the accurate quantitative data of the decision problem best. The $\mathrm{V}$-shape transformation function requires accurate quantitative data such as operational and technical data. All measurements performed for this research are considered accurate, even though only one experiment per biopolymer option is executed. Most experiments are not repeated, so that the variability of the measurements are not quantifiable. That is why the measurements and conclusions can only be interpreted as indicative. In the case of the total amount of readily separable chemicals, the Usual transformation function is applicable, because only two nominal values exist: ' 0.00 ' or ' 29.70 '.

In the case of the V-shape transformation function, the preference degree increases linearly until the deviation between the evaluations of two biopolymer options on a single criterion reaches the preference threshold $p$ [13-15], please refer to Fig. 4. In order to minimise the loss of information no indifference threshold $q$ is defined. Here, it is assumed that even the smallest difference stimulates some kind of preference over the other. However, a very small preference is achieved. The preference threshold $p$ is considered as the lowest value above which there is strict preference $\left(P_{i}(a, b)=1\right)$ of one of the corresponding biopolymer options over the other. It should be noted that the preference threshold $p$ is defined as a function of the deviation between the evaluations on the criteria and not as a function of the evaluation

Table 5

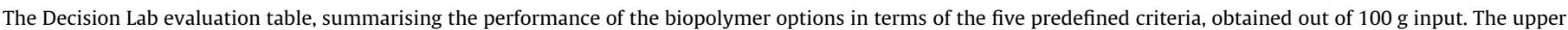
rows contain the requested preference parameters, while the lower rows describe the eight biopolymer options. The columns represent the five predefined criteria.

\begin{tabular}{|c|c|c|c|c|c|}
\hline & Water-free bio-oil & Water content & Energy recuperation & Char & Separable chemicals \\
\hline Min/Max & Maximise & Minimise & Maximise & Minimise & Maximise \\
\hline Weight & 0.2511 & 0.0887 & 0.1525 & 0.0533 & 0.4543 \\
\hline Transformation function & V-shape & V-shape & V-shape & V-shape & Usual \\
\hline Indifference threshold $q$ & - & - & - & - & - \\
\hline Preference threshold $p$ & 14.89 & 21.12 & 27.9 & 12.89 & - \\
\hline Gaussian threshold $s$ & - & - & - & - & - \\
\hline Threshold unit & $\mathrm{g}$ & $\mathrm{m} \%$ & $\%$ & $\mathrm{~g}$ & $\mathrm{~g}$ \\
\hline Average performance & 38 & 22.48 & 50.4 & 14.42 & 3.71 \\
\hline S.D. & 6.13 & 8.47 & 8.3 & 3.64 & 10.5 \\
\hline Unit & $\mathrm{g}$ & $\mathrm{m} \%$ & $\%$ & $\mathrm{~g}$ & $\mathrm{~g}$ \\
\hline Willow (reference) & 31.74 & 36.65 & 43.1 & 22.39 & 0.00 \\
\hline Willow/PLA 1:1 & 43.89 & 15.53 & 51.5 & 13.46 & 0.00 \\
\hline Willow/corn starch $1: 1$ & 31.94 & 26.94 & 38.9 & 14.47 & 0.00 \\
\hline Willow/PHB $1: 1$ & 29.03 & 15.97 & 66.8 & 9.5 & 29.70 \\
\hline Willow/Biopearls 1:1 & 43.92 & 16.81 & 51.5 & 12.92 & 0.00 \\
\hline Willow/Eastar 1:1 & 40.53 & 18.96 & 46.3 & 13.92 & 0.00 \\
\hline Willow/Solanyl 1:1 & 39.8 & 32.82 & 52.1 & 15.24 & 0.00 \\
\hline Willow/potato starch $1: 1$ & 43.19 & 16.17 & 52.6 & 13.49 & 0.00 \\
\hline
\end{tabular}



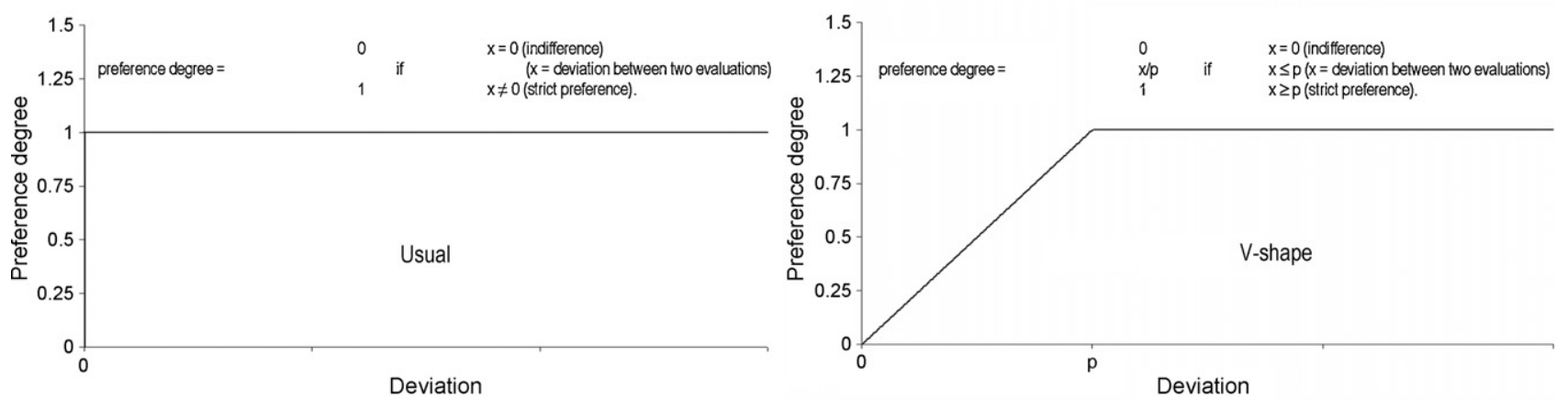

Fig. 4. The Usual and V-shape transformation functions.

itself. For this research, $p$ is set standard at the maximum deviation of each criterion, which is considered the best in order to minimise the loss of information. At $p$, the preference degree takes value 1 , indicating strict preference. Accordingly, the overall (multicriteria) preference index $\pi(a, b)$ of a biopolymer option with regard to the other biopolymer options is obtained by calculating the weighted $\left(w_{i}\right)$ average of all preference degrees $P_{i}(a, b)$ of that specific biopolymer option (Eq. (2)). The closer this value is to 1 , the greater the overall preference is [13-15].

$T T(a, b)=\frac{\sum_{i=1}^{n} w_{i} P_{i}(a, b)}{\sum_{i=1}^{n} w_{i}}, \quad$ with $n=$ the number of criteria.

In addition, preference flows are computed in PROMETHEE and Decision Lab to summarise the results of all pairwise comparisons. The "positive flow" $\Phi^{+}$of a biopolymer option is the preference degree with which this biopolymer option is preferred on average over the other ones. The larger the $\Phi^{+}$, the better the biopolymer option is. Additionally, the "negative flow" $\Phi^{-}$of a biopolymer option is the preference degree with which the other biopolymer options are preferred on average to that biopolymer option. The smaller the $\Phi^{-}$, the better the biopolymer option is. Both $\Phi^{+}$and $\Phi^{-}$do have positive values. Eqs. (3) and (4) represent the mathematical formulation of $\Phi^{+}$and $\Phi^{-}$. Finally, the net flow $\Phi$, also called Phi score, of a biopolymer option is the balance between
$\Phi^{+}$and $\Phi^{-}\left(\Phi=\Phi^{+}-\Phi^{-}\right)$. The larger the net flow, the better the biopolymer option is. Both $\Phi^{+}$and $\Phi^{-}$are used to rank the biopolymer options partially (PROMETHEE I); while the net flow $\Phi$ is used to rank them completely (PROMETHEE II) [13-15].

$$
\phi^{+}=\sum_{\substack{n \in K \\ b \neq a}} T T(a, b)
$$

with $K$ being the set of biopolymer options.

$$
\phi^{-}=\sum_{\substack{n \in K \\ b \neq a}} T T(b, a) .
$$

The PROMETHEE I partial ranking is defined as the intersection of the $\Phi^{+}$and $\Phi^{-}$rankings. It only contains preferences that are confirmed by both preference flows: a biopolymer option is preferred over another only if that biopolymer option has a better (higher) $\Phi^{+}$and a better (lower) $\Phi^{-}$. In case both flows are in conflict, actions are considered incomparable. The preferences that appear in the PROMETHEE I ranking can thus be considered as well established. The PROMETHEE II complete ranking ranks all biopolymer options from the best to the worst, leaving no incomparable pair of biopolymer options. It is directly based on

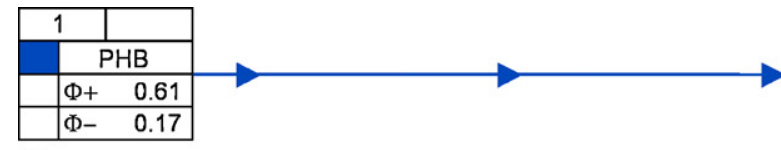

(I)

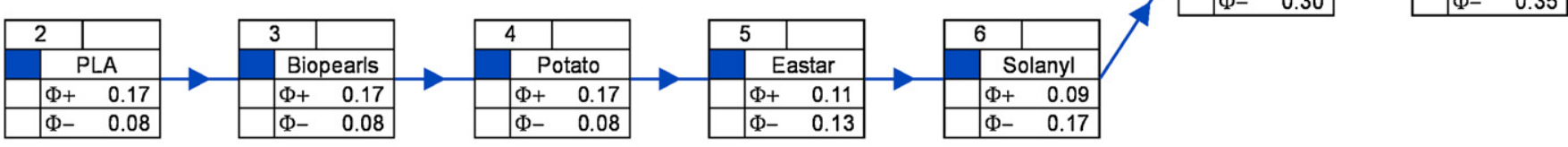

(II)

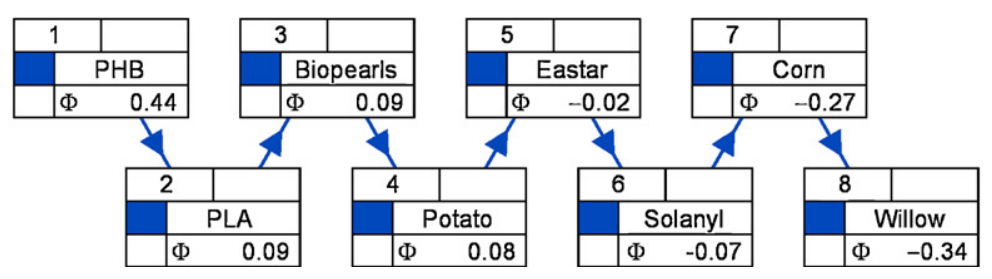

Fig. 5. PROMETHEE I and II ranking of the MCDM problem. 


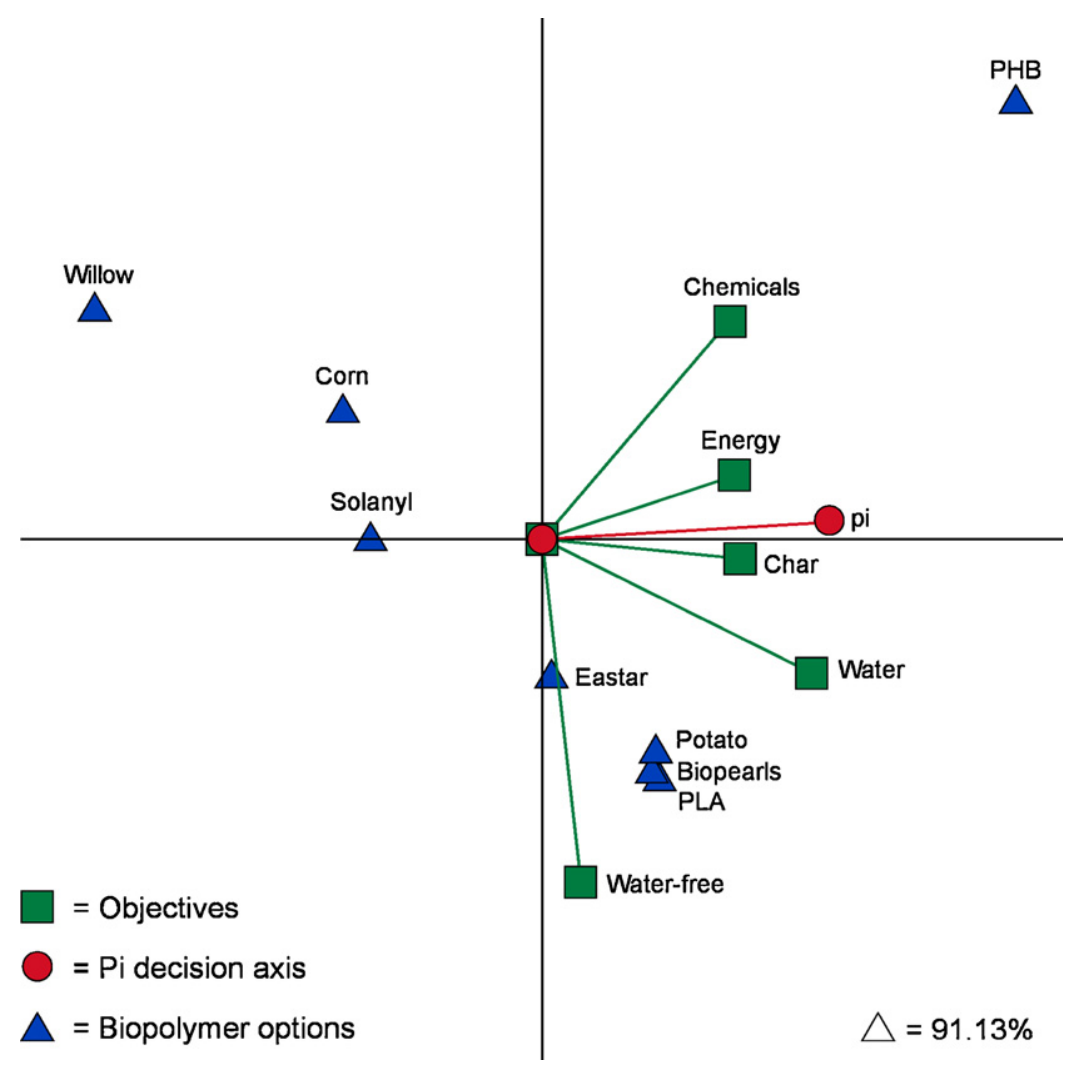

Fig. 6. The GAIA plane of the decision problem.

the net flow $\Phi[13-15]$. Fig. 5 shows the PROMETHEE I and II ranking of the decision problem.

The PROMETHEE I partial ranking shows that the biopolymer options PHB, PLA, Biopearls, potato starch, Eastar and Solanyl are preferred over corn starch and willow; and that corn starch is preferred over willow. It also indicates that PLA is preferred over Biopearls, Biopearls over potato starch, potato starch over Eastar, and Eastar over Solanyl. A slight preference of PLA over Biopearls and of Biopearls over potato starch appears in Fig. 5, even though the three $\Phi^{+}$and $\Phi^{-}$equal 0.17 and 0.08 , respectively, due to rounding errors. Finally, PHB is in conflict with PLA, Biopearls, potato starch, Eastar, and Solanyl: PHB definitely has the highest $\Phi^{+}$, but PLA, Biopearls, potato starch and Eastar have a lower (better) $\Phi^{-}$, while the $\Phi^{-}$of Solanyl is equal (only slightly lower/ better) to the one of PHB. PHB has a relatively high $\Phi^{-}$because it results in the lowest water-free bio-oil yield, which is the second most important criterion of the decision problem (Tables 2 and 4). However, PROMETHEE II undoubtedly prefers PHB over all other biopolymer options because it has the highest net flow $\Phi$. PLA, Biopearls and potato starch have a comparable net flow $\Phi$, with PLA having a slightly higher (better) net flow $\Phi$.

Additionally, Decision Lab provides a descriptive complement of both PROMETHEE rankings: the GAIA plane. The information relative to a decision problem including $k$ criteria can be represented in a $k$-dimensional space. The GAIA plane is obtained by projection of this information on a plane such that as few information as possible is lost. GAIA makes use of the Principal Components Analysis (PCA) method and is applied on the net preference flow $\left(\Phi=\Phi^{+}-\Phi^{-}\right)$. The GAIA plane corresponds to the first two principal components, which ensures that a maximum quantity of information is available in the plane. However, some information usually gets lost in the projection process in the GAIA plane. For this, GAIA generates a $\Delta$ value which serves as a quality control measure. It measures the amount of information preserved in the GAIA plane. In practice, $\Delta$ values larger than $70 \%$ correspond to reliable GAIA planes; $\Delta$ values lower than $60 \%$ should be considered with care [15]. Fig. 6 shows the GAIA plane of the decision problem and has a $\Delta$ value of over $91 \%$. In the GAIA plane the criteria are presented by axes connected to squares, and the biopolymer options by triangular shapes. The weights of the criteria are represented by a separate axis, called the Pi decision axis. This decision axis shows the kind of compromise solution that is proposed by PROMETHEE.

The GAIA plane confirms the observations from the PROMETHEE rankings. PHB is the biopolymer option that is in best agreement with the Pi decision axis and is especially supported by

Table 6

Weight sensitivity analysis of the decision problem.

\begin{tabular}{|c|c|c|c|c|c|c|c|}
\hline \multirow[t]{2}{*}{ Objective } & \multicolumn{4}{|c|}{ Absolute values } & \multicolumn{3}{|c|}{ Relative values (\%) } \\
\hline & Unit & Weight & Min & Max & Weight & Min & Max \\
\hline Water-free bio-oil yield & $\mathrm{g}$ & 0.2511 & 0.1294 & 0.5536 & $25.11 \%$ & $14.73 \%$ & $42.50 \%$ \\
\hline Water content & $\mathrm{m} \%$ & 0.0887 & 0.0452 & 0.2856 & $8.87 \%$ & $4.73 \%$ & $23.86 \%$ \\
\hline Energy recuperation & $\%$ & 0.1525 & 0.000 & 0.3038 & $15.25 \%$ & $0.00 \%$ & $26.39 \%$ \\
\hline Char yield & $\mathrm{g}$ & 0.0533 & 0.000 & 0.1162 & $5.33 \%$ & $0.00 \%$ & $10.94 \%$ \\
\hline Yield in readily separable chemicals & $\mathrm{g}$ & 0.4543 & 0.1524 & Infinity & $45.43 \%$ & $21.84 \%$ & $100.00 \%$ \\
\hline
\end{tabular}




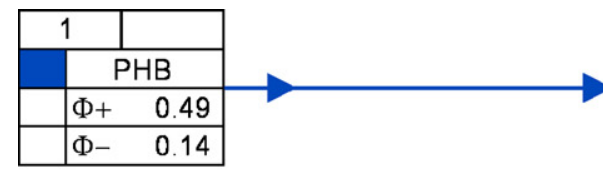

(I)

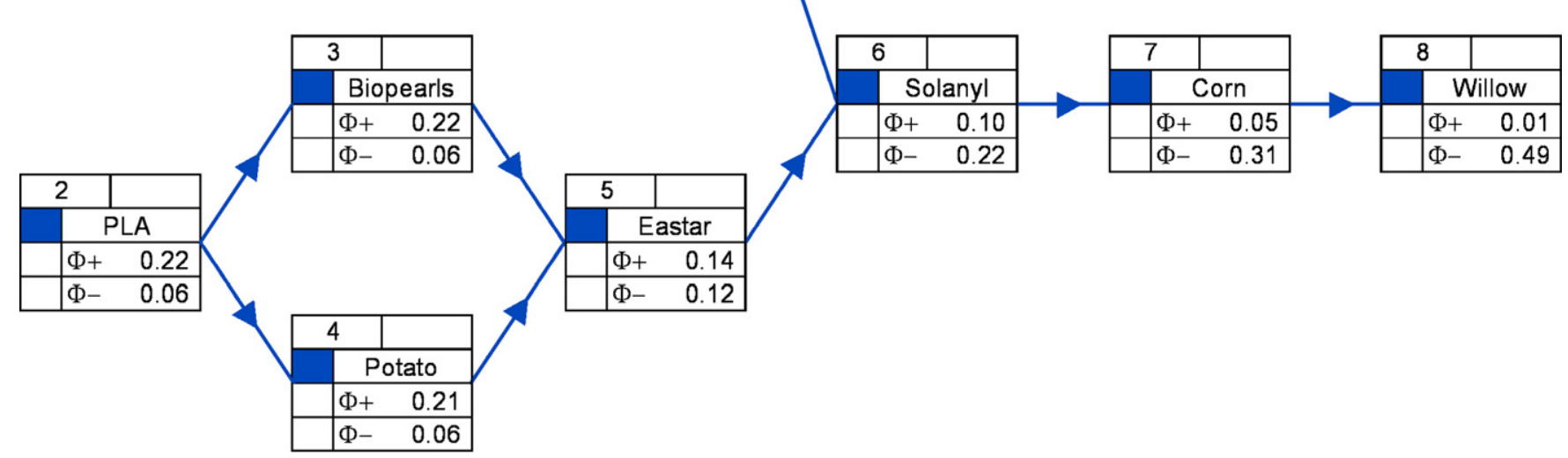

(II)

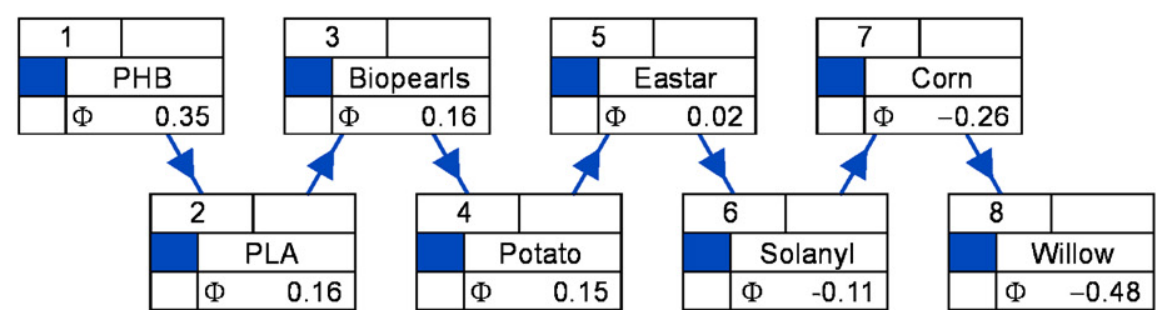

Fig. 7. PROMETHEE I and II ranking of the equal weights scenario.

the criteria readily separable chemicals and energy recuperation. PLA, Biopearls and potato starch are clustered, indicating that these biopolymer options have similar profiles. Out of these three biopolymer options, PLA is located the furthest in the direction of the Pi decision axis and is thus considered as the best option. Eastar seems to "flirt" with the borders. Solanyl, corn starch and willow are directed towards the opposite direction of the Pi decision axis, with Solanyl the closest and willow the furthest. The GAIA plane also indicates that the total amount of readily separable chemicals and the water-free bio-oil yield are the two most conflicting criteria.

Finally, a weight sensitivity analysis is performed. Table 6 summarises the range within which the respective weights of each criterion are allowed to deviate (ceteris paribus) without changing the PROMETHEE II ranking of all the biopolymer options. It can be observed that the decision problem is fairly robust: the weights of each criterion can be altered in a relatively wide range, without any further consequence. The char yield presents the most narrow interval. However, even if the char yield would double in importance, which for this research is unlikely, no alterations in the PROMETHEE II ranking are induced.

Up till now, all conclusions have been based upon a single scenario of the decision problem: the base scenario. In order to investigate the robustness of the methodology applied to a full extent, a second scenario will additionally be discussed: the scenario based on equal weights. In this approach, all criteria are assumed to be of equal importance $\left(w_{i}=0.2\right)$. All other parameters of the base scenario remain unchanged. Fig. 7 shows that the PROMETHEE II ranking is completely identical to that in Fig. 5. Clear differences in the net flow $\Phi$ are observed, however.
Moreover, the PROMETHEE I ranking shows a different pattern as compared to the base scenario (Fig. 5). Here, the biopolymer options PHB, PLA, Biopearls, potato starch and Eastar are preferred over Solanyl, corn starch and willow; and Solanyl is preferred over corn starch, and corn starch over willow. Thus, Solanyl slightly decreased in the level of preference. PLA is still preferred over Biopearls, potato starch and Eastar. But, Biopearls is now considered as incomparable with potato starch: Biopearls has a higher $\Phi^{+}$, while potato starch has a slightly lower (better) $\Phi^{-}$. Relatively, potato starch slightly increased in the level of preference. Finally, both Biopearls and potato starch are still preferred over Eastar.

Taking into account the five predefined criteria, it can be concluded that the flash co-pyrolysis of biomass and biopolymers results in improved pyrolysis characteristics. PHB is always preferred over PLA, and PLA is preferred over all the other biopolymer options. However, PLA, Biopearls and potato starch are almost identical. Additionally, PHB, PLA, Biopearls, potato starch and Eastar are always preferred over Solanyl, corn starch and willow. In both scenarios, the first four biopolymer options of the PROMETHEE II ranking are similar to the ones that resulted in a synergy earlier (Table 3 ).

\section{Critical remark}

For water determination the Dean Stark method has been applied. Due to the fact that some polar components are stripped together with the water (steam distillation), the distilled water will contain some impurities, resulting in an overestimation of the respective water content. Therefore, the water phase separated 
from willow bio-oil has been additionally investigated on $\mathrm{pH}$, neutralisation number and composition. $\mathrm{pH}$ is measured with a glass calomel electrode and neutralisation is performed by a potentiometric titration with $0.02 \mathrm{~N} \mathrm{NaOH}$ (ASTM D 1067). Finally, the composition of the distilled water is investigated by GC/MS. Based on the additional experiments, it can be calculated that the water determinations for willow bio-oil are overestimated by around $10 \%$, which is mainly caused by acetic acid and minor amounts of formic acid. If the overestimation of $10 \%$ is generally considered, the observations towards the occurrence of a synergy and the pairwise comparisons described above remain indifferent.

However, the amount of acidic and formic acid in the bio-oils originating from the willow/biopolymer blends is fairly lower, thereby inducing smaller overestimations of the respective water content. The minimal level of overestimation will be $5 \%$ for each $1: 1$ blend (50\% of the willow overestimation). In the case of an overestimation of $5 \%$ (only induced by the willow fraction) which is considered as the worst case scenario considering its impact on the previous observations, again no crucial implications occur: the occurrence of a synergy is still indicated for the $1: 1$ blends of willow/PLA, willow/PHB and willow/potato starch (for 1:1 willow/ Biopearls the indication towards the occurrence of a synergy is not straightforward anymore). Additionally, the pairwise comparisons of all biopolymer options against each other remain practically unchanged. Willow slightly becomes more interesting, but still results in the second worst amount of pyrolytic water.

When higher overestimations (between $5 \%$ (pessimistic scenario) and $10 \%$ (optimistic scenario)) occur for the blends, the observations will only become more similar to the observations previously described. Therefore, it can be stated that the choice of water determination and the small overestimations of the water content do not result in significant implications, misinterpretations or wrong conclusions.

\section{Conclusions}

With the readily separation of chemicals and the water-free bio-oil yield as the most important criteria, the flash co-pyrolysis of biomass and biopolymers is a promising route to produce energy and materials in a sustainable manner and can be regarded as a win-win situation, which can easily be converted into an economically attractive industrial process. Flash co-pyrolysis generally results in bio-oil with a reduced water content, an enhanced pyrolysis yield, a reduction of the waste volume, and a more attractive recycling route for biopolymers. This allows the flash co-pyrolysis of willow and biopolymers to be defined as an interesting upgrading step for the pyrolysis of biomass waste streams, a supplier of value-added materials and renewable energy, and as an alternative waste treatment option. Even though all 1:1 willow/biopolymer blends result in improved pyrolysis characteristics at $723 \mathrm{~K}$ as compared with pure willow, polyhydroxybutyrate, polylactic acid, Biopearls and potato starch are the most performant options. These four biopolymers additionally result in a synergy during co-pyrolysis with willow: a decrease in the amount of pyrolytic water, higher than theoretically expected, is observed.

\section{Acknowledgments}

The authors would like to thank the many suppliers of the biopolymer samples: Biomer, Luts Plastic Production, Rodenburg Biopolymers, Biopearls, and Jemaco. The authors would also like to show their gratitude towards Frédéric Hallot for providing us with 'MCDM tool'. This work fits into an association scholarship between Hasselt University and Xios.

\section{References}

[1] A. Demirbas, G. Arin, Energy Sources 24 (2002) 471.

[2] B.V. Babu, A.S. Chaurasia, Energy Conv. Manag. 44 (2003) 2135.

[3] O. Onay, O.M. Kockar, Renew. Energy 28 (2003) 2417.

[4] S. Czernik, A.V. Bridgwater, in: Bridgwater (Ed.), Fast Pyrolysis of Biomass: A Handbook, vol. 3, CPL Press, United Kingdom, 2005, p. 105.

[5] A.V. Bridgwater, G.V.C. Peacocke, Renew. Sust. Energy Rev. 4 (2000) 1.

[6] V.A. Doshi, H.B. Vuthaluru, T. Bastow, Fuel Process. Technol. 86 (2005) 885.

[7] D. Mohan, C.U. Pittman, P.H. Steele, Energy Fuels 20 (2006) 848.

[8] A. Oasmaa, C. Peacocke, S. Gust, D. Meier, R. McLellan, Energy Fuels 19 (2005) 2155.

[9] T. Cornelissen, J. Yperman, G. Reggers, S. Schreurs, R. Carleer, Fuel 87 (2008) 1031.

[10] T. Cornelissen, M. Jans, J. Yperman, G. Reggers, S. Schreurs, R. Carleer, Fuel 87 (2008) 2523.

[11] C. Lievens, J. Yperman, J. Vangronsveld, R. Carleer, Fuel 87 (2008) 1894.

[12] C. Lievens, J. Yperman, T. Cornelissen, R. Carleer, Fuel 87 (2008) 1906.

[13] J.P. Brans, P.H. Vincke, Manag. Sci. 31 (1985) 647.

[14] J.P. Brans, P. Vincke, B. Mareschal, Eur. J. Oper. Res. 24 (1986) 228.

[15] Decision Lab 2000, Executive Edition, Getting Started Guide, Visual Decision Inc., 1999, p. 66.

[16] T.L. Saaty, J.M. Alexander, Biol. Soc. Sci. (1981) 148.

[17] M. Deschoenmakere, Inventarisatie en analyse van verschillende materiaal- en afvalaspecten van bioplastics, Ghent Univeristy, Gent, 2007, p. 131.

[18] V. Massardier-Nageotte, C. Pestre, T. Cruard-Pradet, R. Bayard, Polym. Degrad. Stab. 91 (2006) 620.

[19] M. Jans, Co-pyrolyse van biomassa met biopolymeren ter reductie van het watergehalte in bio-olie, Dep. IWT, Diepenbeek, 2007, p. 190.

[20] http://www.biomer.de.

[21] http://www.hyacail.com.

[22] http://www.biopearls.nl.

[23] http://www.verpakkingsmanagement.nl.

[24] P. De Beir, F. Hallot, H. Pastijn, MCDM Tool 1.0, Beta Version, Eliseo, 1998-2005. 